\title{
A Hopf Operad of Forests of Binary Trees and Related Finite-Dimensional Algebras
}

\author{
FRÉDÉRIC CHAPOTON \\ Institut Girard Desargues, Université Lyon 1, 21 Avenue Claude Bernard, F-69622 Villeurbanne Cedex, France
}

Received September 18, 2002; Revised October 1, 2003; Accepted October 23, 2003

Abstract. The structure of a Hopf operad is defined on the vector spaces spanned by forests of leaf-labeled,
rooted, binary trees. An explicit formula for the coproduct and its dual product is given, using a poset on forests.

Keywords: Hopf operad, binary tree, poset

\section{Introduction}

The theme of this paper is the algebraic combinatorics of leaf-labeled rooted binary trees and forests of such trees. We shall endow these objects with several algebraic structures.

The main structure is an operad, called the Bessel operad, which is the suspension of an operad defined by a distributive law between the suspended commutative operad and the operad of commutative non-associative algebras (sometimes called Griess algebras). The Bessel operad may be seen as an analog of the Gerstenhaber operad [10], which is the suspension of an operad defined by a distributive law between the suspended commutative operad and the Lie operad. Unlike the Gerstenhaber operad, the Bessel operad has a simple combinatorial basis, given explicitly by forests of leaf-labeled rooted binary trees.

The Bessel operad, like the Gerstenhaber operad, is a Hopf operad. More precisely, they are both endowed with a cocommutative coproduct. This gives rise to a family of finitedimensional coalgebras. In the dual vector spaces of the Bessel operad, one gets algebras based on forests of leaf-labeled binary trees.

An explicit formula is obtained for the coproduct in these coalgebras of forests (and therefore for their dual products), using a poset structure on the set of forests, which may be of independent interest.

After some preliminary material on operads in the first section, the second section is devoted to the definition of a distributive law between the suspended commutative operad and the Griess operad. The suspension of the operad defined by this distributive law is introduced in the next section. The coproduct is defined and shown to be given by an explicit sum in the fourth section. In the last section, the dual algebras are briefly studied. 


\section{Generalities on operads}

As the usual setup for operads $[4,8,9]$ is slightly different from the way operads are dealt with here, this section gathers some conventions and definitions.

An operad $\mathcal{P}$ is seen as a functor from the groupoid of finite sets and bijections to some symmetric monoidal category (vector spaces for example) together with binary composition maps satisfying some natural axioms. If the target category is the category of sets, the underlying functor is a species in the sense of [2].

Finite sets will be denoted by capital letters $I, J, K, \ldots$ Elements of finite sets will be denoted by letters $i, j, k, \ldots$. The symbols $\star$ and \# are used as place-holders for composition maps.

The composition map $\circ_{\star}$ is defined for any two finite sets $I$ and $J$ as a map from $\mathcal{P}(I \sqcup\{\star\}) \otimes \mathcal{P}(J)$ to $\mathcal{P}(I \sqcup J)$. Other symbols such as \# are used instead of $\star$ when iterated compositions appear.

The tensor product $\otimes$ on the category of operads is given on the level of functors by $(\mathcal{P} \otimes \mathcal{Q})(I)=\mathcal{P}(I) \otimes \mathcal{Q}(I)$ and by the tensor products of composition maps.

A presentation by generators and relations of an operad is given as follows: some generators labelled by their inputs, with some specific symmetry properties with respect to the symmetric group on these inputs, and some relations involving compositions of these generators.

Under some mild hypothesis on the target category, there is a monoidal structure on the category of functors starting from the groupoid of finite sets, which is called the composition product and denoted by $\circ$. Then an operad can equivalently be defined as a monoid for $\circ$. In this context, a distributive law relating two operads $\mathcal{P}$ and $\mathcal{Q}$ is a morphism of functors from $\mathcal{P} \circ \mathcal{Q}$ to $\mathcal{Q} \circ \mathcal{P}$ which induces an operad structure on $\mathcal{Q} \circ \mathcal{P}$. For details on this notion, see [7].

To describe a distributive law between two operads given by generators and relations, it is sufficient to define it on single compositions of generators. Then a consistency condition has to be checked on the double compositions of generators, see [7, Section 2] for more on this.

\section{A distributive law}

All the operads considered here are in the monoidal category of complexes of vector spaces over $\mathbb{Q}$ with zero differential, i.e. the category of vector spaces over $\mathbb{Q}$ which are graded by $\mathbb{Z}$, with Koszul sign rules for the tensor product. An Hopf operad is an operad $\mathcal{P}$ with a coassociative morphism of operads from $\mathcal{P}$ to $\mathcal{P} \otimes \mathcal{P}$.

A tree is a leaf-labeled rooted binary tree and a forest is a set of such trees, see figure 1. Vertices are either inner vertices (valence 3 ) or leaves and roots (valence 1 ). By convention, edges are oriented towards the root. Leaves are bijectively labeled by a finite set. A half-edge is a pair made of an inner vertex and an incident edge (incoming or outgoing). Trees and forests are pictured with their roots down and their leaves up, but are not to be considered as planar. 


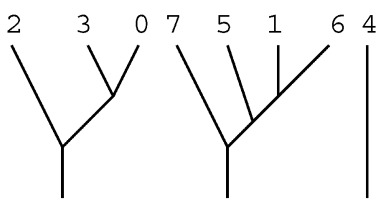

Figure 1. A forest on $\{0,1,2, \ldots, 7\}$.

\subsection{The determinant operad and orientations}

An orientation of a finite set $X$ is a generator of the $\mathbb{Z}$-module $\Lambda^{|X|} \mathbb{Z} X$. For example, $1 \wedge 3 \wedge 4 \wedge 2$ is an orientation of $\{1,2,3,4\}$.

Let us recall the definition of the suspended commutative associative operad Det introduced by Ginzburg and Kapranov [4]. Let $I$ be a finite set. The vector space $\operatorname{Det}(I)$ is the determinant vector space $\Lambda^{|I|} \mathbb{Q} I$ placed in degree $|I|-1$. Any orientation of $I$ gives a basis of $\operatorname{Det}(I)$. The composition of the operad Det is given by the rule

$$
(x \wedge \star) \circ_{\star} y=x \wedge y,
$$

for all $x \in \operatorname{Det}(I)$ and $y \in \operatorname{Det}(J)$.

It is well known and easy to check that Det has the presentation by the antisymmetric generator $e_{i, j}=i \wedge j$ of degree 1 in $\operatorname{Det}(\{i, j\})$ satisfying

$$
e_{i, \star} \circ_{\star} e_{j, k}=e_{k, \star} \circ_{\star} e_{i, j}
$$

The operad Det is binary quadratic and Koszul, see [4] for the definitions of these notions.

\subsection{The Griess operad and rooted binary trees}

The operad Gri describing commutative but not necessarily associative algebras (sometimes called Griess algebras) admits the following description. The space Gri( $I)$ has a basis indexed by rooted binary trees with leaves labeled by $I$ and the composition is grafting. This vector space is placed in degree 0 . In fact, Gri is the free operad on a binary symmetric generator $\omega_{i, j}$ of degree 0 corresponding to the unique rooted binary tree with two leaves labeled by $\{i, j\}$. The operad Gri is binary quadratic and Koszul.

\subsection{The operad B of root-oriented forests}

Proposition 2.1 The following formula defines a distributive law from Gri $\circ$ Det to Det $\circ$ Gri:

$$
\omega_{i, \star} \circ_{\star} e_{j, k}=e_{j, \star} \circ_{\star} \omega_{i, k}-e_{k, \star} \circ_{\star} \omega_{i, j}
$$


Proof: As Gri is a free operad, one has only to check that the rewriting of

$$
\omega_{i, \star} \circ_{\star}\left(e_{j, \#} \circ_{\#} e_{k, \ell}\right)-\omega_{i, \star} \circ_{\star}\left(e_{k, \#} \circ_{\#} e_{\ell, j}\right),
$$

using (3) as a replacement rule, gives zero modulo the relation (2) which defines Det. Indeed, one has

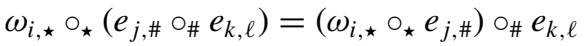

$$
\begin{aligned}
& =\left(e_{j, \star} \circ_{\star} \omega_{i, \#}-e_{\#, \star} \circ_{\star} \omega_{i, j}\right) \circ_{\#} e_{k, \ell} \\
& =e_{j, \star} \circ_{\star}\left(\omega_{i, \# O_{\#}} e_{k, \ell}\right)-\left(e_{\#, \star} \circ_{\star} \omega_{i, j}\right) \circ_{\#} e_{k, \ell} \\
& =e_{j, \star} \circ_{\star}\left(e_{k, \# O_{\#}} \omega_{i, \ell}-e_{\ell, \# \circ_{\#}} \omega_{i, k}\right)-\left(e_{\#, \star} \circ_{\#} e_{k, \ell}\right) \circ_{\star} \omega_{i, j}
\end{aligned}
$$

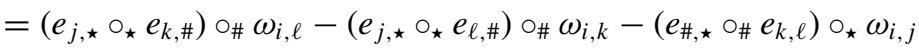

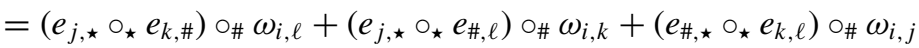

$$
\begin{aligned}
& =\left(e_{j, \star} \circ_{\star} e_{k, \#}\right) \circ_{\#} \omega_{i, \ell}+\left(e_{\ell, \star} \circ_{\star} e_{j, \#}\right) \circ_{\#} \omega_{i, k}+\left(e_{k, \star} \circ_{\star} e_{\ell, \#}\right) \circ_{\#} \omega_{i, j} \text {. }
\end{aligned}
$$

This expression is invariant by cyclic permutations of $j, k, \ell$. This shows that the rewriting of (4) is zero, which proves the proposition.

Let us summarize the description of the operad defined by this distributive law.

Proposition 2.2 The operad B defined on Det $\circ$ Gri by this distributive law is isomorphic to the quotient of the free operad generated by $e_{i, j}$ antisymmetric in degree 1 and $\omega_{i, j}$ symmetric in degree 0 by the following relations.

$$
\begin{aligned}
e_{i, \star} \circ_{\star} e_{j, k} & =e_{k, \star} \circ_{\star} e_{i, j}, \\
\omega_{i, \star} \circ_{\star} e_{j, k} & =e_{j, \star} \circ_{\star} \omega_{i, k}-e_{k, \star} \circ_{\star} \omega_{i, j} .
\end{aligned}
$$

Corollary 2.3 The operad B is binary quadratic and Koszul.

Proof: Koszulness follows from a theorem of Mark1 [7] since $B$ is defined by a distributive law between two Koszul operads.

A root-orientation of a forest $F$ is an orientation of the set of roots of $F$. A root-oriented forest is a tensor product of a root-orientation and a forest, see figure 2 . By the construction of $B$ by a distributive law, the vector space $B(I)$ has a basis indexed by root-oriented forests. The degree of a root-oriented forest is the number of roots minus one.

Here is a partial description of the composition, in the case where the first element is a generator. Let $F_{1} \sqcup F_{2}$ be the disjoint union of two forests $F_{1}$ and $F_{2}$. We use (from now on) the abuse of notation $(-1)^{x}$ for $(-1)^{\operatorname{deg}(x)}$ when $x$ is homogeneous and also $(-1)^{\circ}$ instead of $(-1)^{\operatorname{deg}(\circ)}$ for any kind of orientation $o$. The degree of an orientation is the number of wedge signs that it contains. The generator $e_{i, j}$ acts on forests by disjoint union in the following sense. 


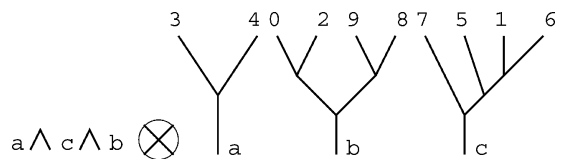

Figure 2. A root-oriented forest on $\{0,1,2, \ldots, 9\}$.

Proposition 2.4 Let $o_{1} \otimes F_{1}$ and $o_{2} \otimes F_{2}$ be two root-oriented forests. Then

$$
e_{\star, \#} \circ_{\star}\left(o_{1} \otimes F_{1}\right) \circ_{\#}\left(o_{2} \otimes F_{2}\right)=(-1)^{o_{1}} o_{1} \wedge o_{2} \otimes\left(F_{1} \sqcup F_{2}\right) .
$$

Proof: The proposition can be restated as follows. Let $x \in B(I)$ and $y \in B(J)$. Then

$$
\left(e_{\star, \#} \circ_{\star} x\right) \circ_{\#} y=(-1)^{x} x \wedge y,
$$

Indeed, one has $e_{\#, \star} \circ_{\star} x=\# \wedge x$ and $(x \wedge \#) \circ_{\#} y=x \wedge y$ by the composition rule of Det. The sign is given by $e_{\#, \star}=-e_{\star, \#}$ and $\# \wedge x=(-1)^{x+1} x \wedge \#$.

Let $T_{1} \vee T_{2}$ be the tree obtained by grafting $T_{1}$ and $T_{2}$ on the two leaves of the tree with one inner vertex. The generator $\omega_{i, j}$ acts on trees by grafting in the following sense.

Proposition 2.5 Let $o_{1} \otimes T_{1}$ and $o_{2} \otimes T_{2}$ be two root-oriented trees. Then

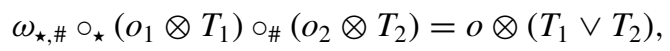

where $o$ is the unique root-orientation of the tree $T_{1} \vee T_{2}$.

Proof: This is just the composition of Gri, restated inside $B$, by definition of the composition in an operad defined by a distributive law.

\section{The Bessel operad as a suspension}

This section is devoted to the operad Bess $=$ Det $\otimes B$ which is a suspended version of $B$. This suspension is necessary for the definition of a Hopf operad structure in the next section. Note that the word "suspension" is just used here to mean the tensor product with Det, even if it corresponds to the usual shift of degree on the level of algebras.

The generating series of the operad Bess has for coefficients the Bessel polynomials $[5,6]$, which are known to count the forests (sets) of rooted leaf-labeled binary trees, hence the chosen name.

\subsection{Outer and inner orientations}

By its definition, the vector space $\operatorname{Bess}(I)$ has a basis indexed by tensor products $o_{1} \otimes o_{2} \otimes F$ where $o_{1}$ is an orientation of $I$ and $o_{2}$ is a root-orientation of the forest $F$. This tensor 
product of two orientations is called an outer orientation of $F$. In this section, an alternative description is given for this kind of orientation, which will be more convenient later.

A global orientation of a forest $F$ is an orientation of the set $V(F) \sqcup\left\{R_{F}\right\}$, where $V(F)$ is the set of inner vertices of $F$ and $R_{F}$ is an auxiliary element.

A local orientation of a forest $F$ at an inner vertex $v$ is an orientation of its 3 incident half-edges (which is of course equivalent to a cyclic order).

An inner-oriented forest is a tensor product $o \otimes \bigotimes_{v \in V(F)} o_{v} \otimes F$, where $o$ is a global orientation of the forest $F$ and the $o_{v}$ are local orientations of $F$ at its inner vertices. This will from now on be abridged $o \otimes F$, where $o$ is a global orientation, the local orientations being implicit. Notice that the order in the product of the local orientations do not matter, as they have degree 2.

One can identify an outer orientation $o_{1} \otimes o_{2}$ with an inner orientation in the following way:

1. Consider the exterior product $o_{1} \wedge R_{F} \wedge o_{2}$ where $R_{F}$ is an auxiliary element.

2. Remove from this exterior product all possible pairs $\ell \wedge r$ where $\ell$ is a leaf and $r$ is a root which are related by an edge.

3. Add to this exterior product pairs $e^{+} \wedge e^{-}$for all edges $e$ between two inner vertices. Here $e^{+}$(resp. $e^{-}$) stands for the upper (resp. lower) half-edge.

The result is an exterior product on all half-edges of $F$ and an auxiliary element $R_{F}$. One can assume that half-edges are gathered by three according to their incident inner vertex. Replacing each such triple $e_{v}^{1} \wedge e_{v}^{2} \wedge e_{v}^{3}$ by the vertex $v$, one gets a global orientation of $F$. One has to keep track of what has been replaced. This is done by assigning the local orientation $o_{v}=e_{v}^{1} \wedge e_{v}^{2} \wedge e_{v}^{3}$ to the inner vertex $v$.

Here is an example of this equivalence of orientations. Consider the outer-oriented forest shown in figure 3 . One can compute the corresponding inner orientation.

$$
\begin{aligned}
1 & \wedge 2 \wedge 4 \wedge 3 \wedge 5 \wedge R_{F} \wedge a \wedge c \wedge b \\
& =1 \wedge 2 \wedge 3 \wedge 5 \wedge R_{F} \wedge a \wedge b \\
& =1 \wedge 2 \wedge 3 \wedge R_{F} \wedge a \\
& =1 \wedge 2 \wedge 3 \wedge R_{F} \wedge a \wedge e^{+} \wedge e^{-} \\
& =\left(1 \wedge 2 \wedge e^{+}\right) \wedge R_{F} \wedge\left(3 \wedge a \wedge e^{-}\right),
\end{aligned}
$$

where $e^{+}$and $e^{-}$are the upper and lower half-edges of the unique inner edge. Hence one

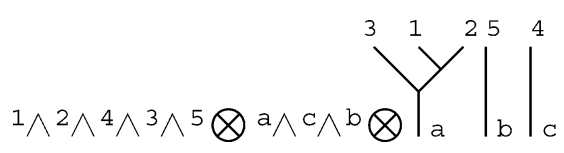

Figure 3. An outer-oriented forest on $\{1,2,3,4,5\}$. 


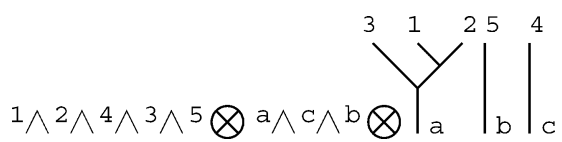

Figure 4. An inner-oriented forest on $\{1,2,3,4,5\}$.

can take the global orientation to be $s \wedge R_{F} \wedge t$ (where $s$ is the upper vertex and $t$ the lower one) and the local orientations to be $1 \wedge 2 \wedge e^{+}$at vertex $s$ and $3 \wedge a \wedge e^{-}$at vertex $t$. The result is shown in figure 4.

The grading is modified (but its parity is not changed) in order that the forests with no inner vertex are in degree 0 , which will be convenient in the next section. From now on, the degree of an inner-oriented forest is the number of its inner vertices.

\subsection{Presentation of Bess}

From the known presentation of $B$, a presentation of Bess by generators and relations is given in this section.

Let $E_{i, j}$ be the inner-oriented forest with two trees on $\{i, j\}$ defined by the outer-oriented formula $E_{i, j}=(j \wedge i) \otimes e_{i, j}$. It is symmetric of degree 0 . As an inner-oriented forest, it is

$$
R \otimes \stackrel{i j}{\mid j}
$$

Let $\Omega_{i, j}$ be the inner-oriented tree on $\{i, j\}$ defined by the outer-oriented formula $\Omega_{i, j}=$ $(i \wedge j) \otimes \omega_{i, j}$. It is antisymmetric of degree 1 . As an inner-oriented tree, it is given by figure 5 .

Proposition 3.1 The operad Bess is isomorphic to the quotient of the free operad on the generators $E_{i, j}$ symmetric of degree 0 and $\Omega_{i, j}$ antisymmetric of degree 1 by the relations

$$
\begin{aligned}
& E_{i, \star} \circ_{\star} E_{j, k}=E_{k, \star} \circ_{\star} E_{i, j}, \\
& \Omega_{i, \star} \circ_{\star} E_{j, k}=E_{j, \star} \circ_{\star} \Omega_{i, k}+E_{k, \star} \circ_{\star} \Omega_{i, j} .
\end{aligned}
$$

Proof: The tensor product by the operad Det acts essentially by changing all the signs. It is well known that the suspended operad has a presentation by similar generators and

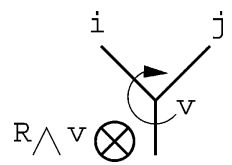

Figure 5. $\Omega_{i, j}$ as an inner-oriented tree. 
relations (up to sign) as it is simply given by a shift of grading at the level of algebras. Let us compute the new relations for our chosen generators. First,

$$
\begin{aligned}
E_{i, \star} \circ_{\star} E_{j, k} & =\left((\star \wedge i) \otimes e_{i, \star}\right) \circ_{\star}\left((k \wedge j) \otimes e_{j, k}\right) \\
& =\left((i \wedge \star) \circ_{\star}(k \wedge j)\right) \otimes\left(e_{i, \star} \circ_{\star} e_{j, k}\right) \\
& =(i \wedge k \wedge j) \otimes\left(e_{i, \star} \circ_{\star} e_{j, k}\right) .
\end{aligned}
$$

Therefore $E_{i, \star} \circ_{\star} E_{j, k}$ is invariant by cyclic permutations of $i, j, k$. One also has

$$
\begin{aligned}
\Omega_{i, \star} \circ_{\star} E_{j, k} & =\left((i \wedge \star) \otimes \omega_{i, \star}\right) \circ_{\star}\left((k \wedge j) \otimes e_{j, k}\right) \\
& =\left((i \wedge \star) \circ_{\star}(k \wedge j)\right) \otimes\left(\omega_{i, \star} \circ_{\star} e_{j, k}\right) \\
& =(i \wedge k \wedge j) \otimes\left(e_{j, \star} \circ_{\star} \omega_{i, k}-e_{k, \star} \circ_{\star} \omega_{i, j}\right) \\
& \left.=(j \wedge i \wedge k) \otimes\left(e_{j, \star} \circ_{\star} \omega_{i, k}\right)+(k \wedge i \wedge j) \otimes\left(e_{k, \star} \circ_{\star} \omega_{i, j}\right)\right) \\
& =E_{j, \star} \circ_{\star} \Omega_{i, k}+E_{k, \star} \circ_{\star} \Omega_{i, j} .
\end{aligned}
$$

Therefore an algebra over Bess is a complex $C$ together with a commutative associative product on $C$ and a commutative not necessarily associative product on the shifted complex $C$ [1], which satisfy a compatibility relation deduced from (11).

The composition inside $E$ is then described as follows.

Proposition 3.2 Let $o_{1} \otimes F_{1}$ and $o_{2} \otimes F_{2}$ be two inner-oriented forests. Then

$$
E_{\star, \#} \circ_{\star}\left(o_{1} \otimes F_{1}\right) \circ_{\#}\left(o_{2} \otimes F_{2}\right)=\left(o_{1} \sqcup o_{2}\right) \otimes\left(F_{1} \sqcup F_{2}\right),
$$

where the global orientation $o_{1} \sqcup o_{2}$ is obtained from $o_{1} \wedge r \wedge o_{2}$ by replacing $R_{1} \wedge r \wedge R_{2}$ by $R$. The local orientations are unchanged.

Proof: Let $o_{1}^{\prime} \otimes o_{1}^{\prime \prime}$ and $o_{2}^{\prime} \otimes o_{2}^{\prime \prime}$ be the corresponding outer orientations of $F_{1}$ and $F_{2}$. Using Proposition 2.4, one has

$$
\begin{aligned}
& E_{\star, \# O_{\star}}\left(o_{1} \otimes F_{1}\right) \circ_{\#}\left(o_{2} \otimes F_{2}\right) \\
& =\left((\# \wedge \star) \otimes e_{\star, \#}\right) \circ_{\star}\left(o_{1}^{\prime} \otimes o_{1}^{\prime \prime} \otimes F_{1}\right) \circ_{\#}\left(o_{2}^{\prime} \otimes o_{2}^{\prime \prime} \otimes F_{2}\right) \\
& =(-1)^{o_{1}^{\prime}+o_{2}^{\prime}+o_{2}^{\prime} o_{1}^{\prime \prime}}\left((\# \wedge \star) \circ_{\star} o_{1}^{\prime} \circ_{\#} o_{2}^{\prime}\right) \otimes\left(e_{\star, \# \circ_{\star}}\left(o_{1}^{\prime \prime} \otimes F_{1}\right) \circ_{\#}\left(o_{2}^{\prime \prime} \otimes F_{2}\right)\right) \\
& =(-1)^{\left(1+o_{1}^{\prime \prime}\right)\left(1+o_{2}^{\prime}\right)} o_{1}^{\prime} \wedge o_{2}^{\prime} \otimes o_{1}^{\prime \prime} \wedge o_{2}^{\prime \prime} \otimes\left(F_{1} \sqcup F_{2}\right) \text {. }
\end{aligned}
$$

Hence the corresponding inner orientation is given by

$$
(-1)^{\left(1+o_{1}^{\prime \prime}\right)\left(1+o_{2}^{\prime}\right)} o_{1}^{\prime} \wedge o_{2}^{\prime} \wedge R \wedge o_{1}^{\prime \prime} \wedge o_{2}^{\prime \prime}
$$

On the other hand, let us compute the orientation corresponding to $o_{1} \sqcup o_{2}$.

$$
o_{1}^{\prime} \wedge R_{1} \wedge o_{1}^{\prime \prime} \wedge r \wedge o_{2}^{\prime} \wedge R_{2} \wedge o_{2}^{\prime \prime}=(-1)^{\left(1+o_{1}^{\prime \prime}\right)\left(1+o_{2}^{\prime}\right)} o_{1}^{\prime} \wedge o_{2}^{\prime} \wedge R \wedge o_{1}^{\prime \prime} \wedge o_{2}^{\prime \prime}
$$

Therefore the two orientations are the same. 
The composition inside $\Omega$ on trees has the following description.

Proposition 3.3 Let $o_{1} \otimes T_{1}$ and $o_{2} \otimes T_{2}$ be two inner-oriented trees. Then

$$
\Omega_{\star, \#} \circ_{\star}\left(o_{1} \otimes T_{1}\right) \circ_{\#}\left(o_{2} \otimes T_{2}\right)=\left(o_{1} \vee o_{2}\right) \otimes\left(T_{1} \vee T_{2}\right),
$$

where the global orientation $o_{1} \vee o_{2}$ is defined by $(-1)^{o_{1}} o_{1} \wedge o_{2}$ modulo $R_{1} \wedge R_{2}=R \wedge v$ where $v$ is the inner vertex of $\Omega$. The local orientations are unchanged.

Proof: Let $o_{1}^{\prime} \otimes \operatorname{root}_{1}$ and $o_{2}^{\prime} \otimes \operatorname{root}_{2}$ be the corresponding outer orientations of $T_{1}$ and $T_{2}$. Using Proposition 2.5, one has

$$
\begin{aligned}
& \left.\Omega_{\star, \#} \circ_{\star}\left(o_{1}^{\prime} \otimes \operatorname{root}_{1} \otimes T_{1}\right) \text { \#\# }_{\left(o_{2}^{\prime}\right.} \otimes \operatorname{root}_{2} \otimes T_{2}\right) \\
& =\left((\star \wedge \#) \circ_{\star} o_{1}^{\prime} \circ_{\#} o_{2}^{\prime}\right) \otimes\left(\omega_{\star, \#} \circ_{\star} \operatorname{root}_{1} \otimes T_{1} \text { ○\# } \operatorname{root}_{2} \otimes T_{2}\right) \\
& =(-1)^{o_{1}^{\prime}}\left(o_{1}^{\prime} \wedge o_{2}^{\prime}\right) \otimes \operatorname{root} \otimes\left(T_{1} \vee T_{2}\right) \text {. }
\end{aligned}
$$

So the corresponding orientation is $(-1)^{o_{1}^{\prime}} o_{1}^{\prime} \wedge o_{2}^{\prime} \wedge R \wedge$ root. Introducing pairs of half-edges gives

$$
(-1)^{o_{1}^{\prime}} o_{1}^{\prime} \wedge o_{2}^{\prime} \wedge R \wedge \operatorname{root} \wedge \operatorname{root}_{1} \wedge e_{1}^{-} \wedge \operatorname{root}_{2} \wedge e_{2}^{-},
$$

where $e_{1}^{-}$and $e_{2}^{-}$are lower half-edges. This is equivalent with the local orientation (root $\wedge$ $e_{1}^{-} \wedge e_{2}^{-}$) at vertex $v$ (which is the local orientation of $\Omega$, see figure 5) and orientation

$$
(-1)^{o_{1}^{\prime}} o_{1}^{\prime} \wedge o_{2}^{\prime} \wedge R \wedge \operatorname{root}_{1} \wedge v \wedge \operatorname{root}_{2}
$$

On the other hand, the proposed orientation is

$$
(-1)^{o_{1}} o_{1}^{\prime} \wedge R_{1} \wedge \operatorname{root}_{1} \wedge o_{2}^{\prime} \wedge R_{2} \wedge \operatorname{root}_{2}=(-1)^{o_{1}} o_{1}^{\prime} \wedge o_{2}^{\prime} \wedge R_{1} \wedge \operatorname{root}_{1} \wedge R_{2} \wedge \operatorname{root}_{2}
$$

This matches the computed orientation, as $R_{1} \wedge R_{2}=R \wedge v$ and $(-1)^{o_{1}}=(-1)^{o_{1}^{\prime}}$.

Let us extend the definition of $\vee$ from trees to forests, as follows. Let $F_{1}=T_{1}^{1} \sqcup T_{1}^{2} \sqcup$ $\cdots \sqcup T_{1}^{m}$ and $F_{2}=T_{2}^{1} \sqcup T_{2}^{2} \sqcup \cdots \sqcup T_{2}^{n}$ be forests, where the $T$ are trees. Define $F_{1} \vee F_{2}$ to be the sum

$$
\sum_{1 \leq a \leq m} \sum_{1 \leq b \leq n}\left(T_{1}^{a} \vee T_{2}^{b}\right) \sqcup T_{1}^{1} \sqcup \cdots \sqcup \hat{T}_{1}^{a} \sqcup \cdots \sqcup T_{2}^{2} \sqcup \cdots \sqcup \hat{T}_{2}^{b} \sqcup \ldots,
$$

where $\hat{T}$ means that this term is absent. In words, $F_{1} \vee F_{2}$ is the sum over all possible pairings of a tree from $T_{1}$ and a tree from $T_{2}$, where these two trees are replaced in the disjoint union $F_{1} \sqcup F_{2}$ by their $\vee$ product. 
Then Proposition 3.3 is still true for forests instead of just trees, with the extended definition just given for $\vee$.

Proposition 3.4 Let $o_{1} \otimes F_{1}$ and $o_{2} \otimes F_{2}$ be two inner-oriented forests. Then

$$
\Omega_{\star, \# \circ_{\star}}\left(o_{1} \otimes F_{1}\right) \circ_{\#}\left(o_{2} \otimes F_{2}\right)=\left(o_{1} \vee o_{2}\right) \otimes\left(F_{1} \vee F_{2}\right)
$$

where the global orientation $o_{1} \vee o_{2}$ is defined by $(-1)^{o_{1}} o_{1} \wedge o_{2}$ modulo $R_{1} \wedge R_{2}=R \wedge v$ where $v$ is the inner vertex of $\Omega$. The local orientations are unchanged.

Proof: By recursion on the total number of trees in $F_{1}$ and $F_{2}$. The proposition is true if $F_{1}$ and $F_{2}$ are trees. Let us assume that $F_{2}$ has at least two trees.

One the one hand,

$$
\begin{aligned}
& \Omega_{\star, \# \circ_{\star}}\left(o_{1} \otimes F_{1}\right) \circ_{\#}\left(\left(o_{2} \sqcup o_{3}\right) \otimes\left(F_{2} \sqcup F_{3}\right)\right) \\
& =\Omega_{\star, \# \circ_{\star}}\left(o_{1} \otimes F_{1}\right) \circ_{\#}\left(E_{\Delta, \infty} \circ_{\Delta}\left(o_{2} \otimes F_{2}\right) \circ_{\infty}\left(o_{3} \otimes F_{3}\right)\right) \\
& =\Omega_{\star, \#} \circ_{\#} E_{\Delta, \infty} \circ_{\star}\left(o_{1} \otimes F_{1}\right) \circ_{\Delta}\left(o_{2} \otimes F_{2}\right) \circ_{\infty}\left(o_{3} \otimes F_{3}\right)
\end{aligned}
$$

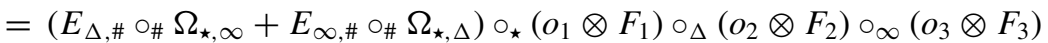

$$
\begin{aligned}
& =(-1)^{o_{2} o_{3}} E_{\Delta, \# O_{\#}} \Omega_{\star, \infty} \circ_{\star}\left(o_{1} \otimes F_{1}\right) \circ_{\infty}\left(o_{3} \otimes F_{3}\right) \circ_{\Delta}\left(o_{2} \otimes F_{2}\right)
\end{aligned}
$$

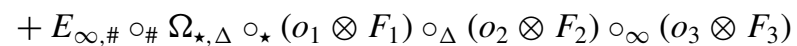

$$
\begin{aligned}
& =(-1)^{o_{2} o_{3}} E_{\Delta, \# \circ_{\#}}\left(\left(o_{1} \vee o_{3}\right) \otimes\left(F_{1} \vee F_{3}\right)\right) \circ_{\Delta}\left(o_{2} \otimes F_{2}\right) \\
& +E_{\infty, \# \circ_{\#}}\left(\left(o_{1} \vee o_{2}\right) \otimes\left(F_{1} \vee F_{2}\right)\right) \circ_{\infty}\left(o_{3} \otimes F_{3}\right) \\
& =(-1)^{o_{2} o_{3}}\left(\left(o_{1} \vee o_{3}\right) \sqcup o_{2}\right) \otimes\left(\left(F_{1} \vee F_{3}\right) \sqcup F_{2}\right) \\
& +\left(\left(o_{1} \vee o_{2}\right) \sqcup o_{3}\right) \otimes\left(\left(F_{1} \vee F_{2}\right) \sqcup F_{3}\right) \text {. }
\end{aligned}
$$

On the other hand, the definition of $\vee$ implies that

$$
\begin{aligned}
& \left(o_{1} \vee\left(o_{2} \sqcup o_{3}\right)\right) \otimes\left(F_{1} \vee\left(F_{2} \sqcup F_{3}\right)\right)=\left(o_{1} \vee\left(o_{2} \sqcup o_{3}\right)\right) \otimes\left(\left(F_{1} \vee F_{3}\right) \sqcup F_{2}\right) \\
& \left.\quad+\left(o_{1} \vee\left(o_{2} \sqcup o_{3}\right)\right) \otimes\left(\left(F_{1} \vee F_{2}\right) \sqcup F_{3}\right)\right) .
\end{aligned}
$$

So it remains to compare the orientations. Using their defining properties, it is easy to see that

$$
(-1)^{o_{2} o_{3}}\left(o_{1} \vee o_{3}\right) \sqcup o_{2}=o_{1} \vee\left(o_{2} \sqcup o_{3}\right)=\left(o_{1} \vee o_{2}\right) \sqcup o_{3}
$$

The proposition is proved.

\section{A coproduct on Bess}

In this section, a map from Bess to Bess $\otimes$ Bess is first defined on generators, then shown to be given by an explicit formula. 


\subsection{Definition on generators}

Let us define a coproduct $\Delta:$ Bess $\rightarrow$ Bess $\otimes$ Bess on the generators $E_{i, j}$ and $\Omega_{i, j}$ of Bess by

$$
\begin{aligned}
& \Delta\left(E_{i, j}\right)=E_{i, j} \otimes E_{i, j}, \\
& \Delta\left(\Omega_{i, j}\right)=E_{i, j} \otimes \Omega_{i, j}+\Omega_{i, j} \otimes E_{i, j} .
\end{aligned}
$$

Proposition 4.1 These formulas define a coassociative cocommutative morphism of operads from Bess to Bess $\otimes$ Bess, i.e. the structure of a Hopf operad on Bess. In particular, each $\operatorname{Bess}(I)$ inherits a structure of cocommutative coalgebra.

Proof: Coassociativity and cocommutativity are clear on generators. One has to check that the relations (10) and (11) of Bess are annihilated by $\Delta$. First,

$$
\Delta\left(E_{i, \star} \circ_{\star} E_{j, k}\right)=\left(E_{i, \star} \otimes E_{i, \star}\right) \circ_{\star}\left(E_{j, k} \otimes E_{j, k}\right)=\left(E_{i, \star} \circ_{\star} E_{j, k}\right) \otimes\left(E_{i, \star} \circ_{\star} E_{j, k}\right),
$$

which inherits the invariance of $E_{i, \star} \circ_{\star} E_{j, k}$ under cyclic permutations of $i, j, k$. Hence $\Delta$ vanishes on the relation (10). For the other relation, on the one hand

$$
\begin{aligned}
\Delta\left(\Omega_{i, \star} \circ_{\star} E_{j, k}\right)= & \left(E_{i, \star} \otimes \Omega_{i, \star}+\Omega_{i, \star} \otimes E_{i, \star}\right) \circ_{\star}\left(E_{j, k} \otimes E_{j, k}\right) \\
= & \left(E_{i, \star} \otimes \Omega_{i, \star}\right) \circ_{\star}\left(E_{j, k} \otimes E_{j, k}\right)+\left(\Omega_{i, \star} \otimes E_{i, \star}\right) \circ_{\star}\left(E_{j, k} \otimes E_{j, k}\right) \\
= & \left(E_{i, \star} \circ_{\star} E_{j, k}\right) \otimes\left(\Omega_{i, \star} \circ_{\star} E_{j, k}\right)+\left(\Omega_{i, \star} \circ_{\star} E_{j, k}\right) \otimes\left(E_{i, \star} \circ_{\star} E_{j, k}\right) \\
= & \left(E_{i, \star} \circ_{\star} E_{j, k}\right) \otimes\left(E_{j, \star} \circ_{\star} \Omega_{i, k}\right)+\left(E_{i, \star} \circ_{\star} E_{j, k}\right) \otimes\left(E_{k, \star} \circ_{\star} \Omega_{i, j}\right) \\
& +\left(E_{j, \star} \circ_{\star} \Omega_{i, k}\right) \otimes\left(E_{i, \star} \circ_{\star} E_{j, k}\right)+\left(E_{k, \star} \circ_{\star} \Omega_{i, j}\right) \otimes\left(E_{i, \star} \circ_{\star} E_{j, k}\right) .
\end{aligned}
$$

On the other hand,

$$
\begin{aligned}
\Delta\left(E_{j, \star} \circ_{\star} \Omega_{i, k}\right) & =\left(E_{j, \star} \otimes E_{j, \star}\right) \circ_{\star}\left(E_{i, k} \otimes \Omega_{i, k}+\Omega_{i, k} \otimes E_{i, k}\right) \\
& =\left(E_{j, \star} \circ_{\star} E_{i, k}\right) \otimes\left(E_{j, \star} \circ_{\star} \Omega_{i, k}\right)+\left(E_{j, \star} \circ_{\star} \Omega_{i, k}\right) \otimes\left(E_{j, \star} \circ_{\star} E_{i, k}\right) \\
& =\left(E_{i, \star} \circ_{\star} E_{j, k}\right) \otimes\left(E_{j, \star} \circ_{\star} \Omega_{i, k}\right)+\left(E_{j, \star} \circ_{\star} \Omega_{i, k}\right) \otimes\left(E_{i, \star} \circ_{\star} E_{j, k}\right),
\end{aligned}
$$

and a similar formula holds for $\Delta\left(E_{k, \star} \circ_{\star} \Omega_{i, j}\right)$. From these formulas, it is clear that $\Delta$ vanishes on relation (11). This proves the proposition.

Remark As it is a coalgebra in the chosen ambient category (see Section 2), the coalgebra structure on $\operatorname{Bess}(I)$ is graded by the number of inner vertices.

\subsection{A poset on forests}

There is an explicit formula for the coproduct, which is a sum over subsets of the set of inner vertices. A poset on forests involved in this formula is described first. 


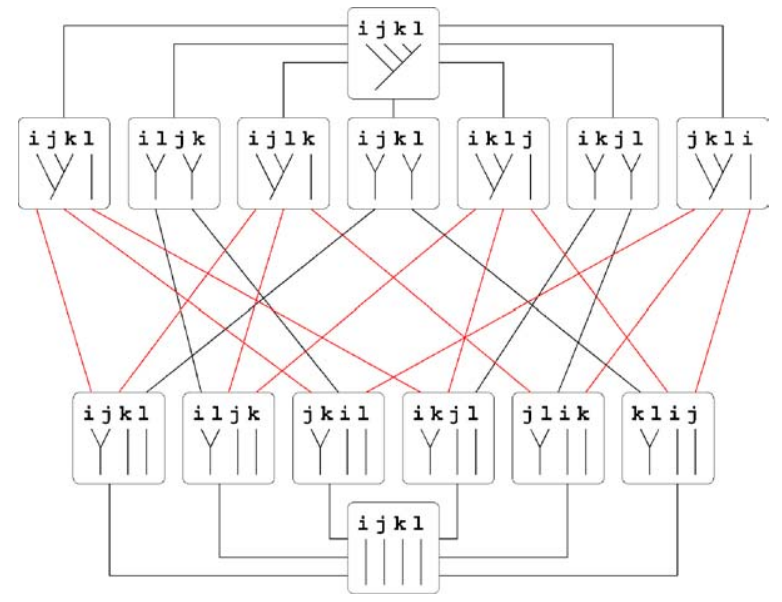

Figure 6. An interval in the poset of forests on $\{i, j, k, \ell\}$.

A leaf is an ancestor of a vertex if there is path from the leaf to the root going through the vertex.

Let $F$ and $F^{\prime}$ be two forests on the set $I$. Then $F^{\prime} \leq F$ if there is a topological map from $F^{\prime}$ to $F$ with the following properties:

1. It is increasing with respect to orientation towards the root.

2. It maps inner vertices to inner vertices injectively.

3. It restricts to the identity on leaves.

In fact, such a topological map from $F^{\prime}$ to $F$ is determined by the image of inner vertices of $F^{\prime}$. Indeed one can recover the map by joining the image of an inner vertex with its ancestor leaves in $F^{\prime}$. See figure 7 for an example of comparable forests, where the topological map is shown using colors.

This relation defines a partial order on the set of forests on $I$. The maximal elements of this poset are the trees. This poset is ranked by the number of inner vertices. Figure 6 displays an interval in the poset of forests on the set $\{i, j, k, \ell\}$.
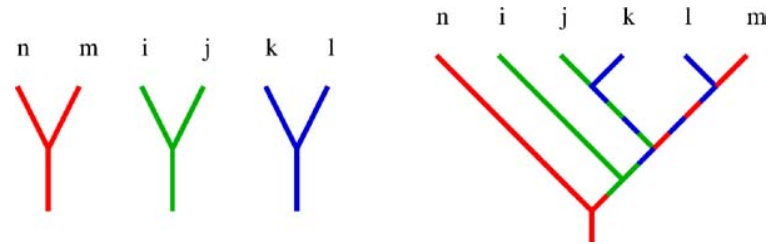

Figure 7. Example for the order relation. 
Remark As can be seen on figure 6, the interval in this poset between the minimal element and one of the "comb" trees (which have a leaf with all the inner vertices belonging to its path to the root) can be identified to the partition lattice. The proof is by identifying a forest with the partition of the set of leaves defined by its trees. Details will be given elsewhere.

If $F$ is a forest on the set $I$ and $V$ is a subset of the set $V(F)$ of inner vertices of $F$, let $\gamma(F, V)$ be the sum of forests $F^{\prime}$ such that $F^{\prime} \leq F$ and the inner vertices of $F^{\prime}$ are identified with the elements of $V$. The sum $\gamma(V, F)$, which is an element of the free $\mathbb{Z}$-module generated by the set of forests on the finite set $I$, can also be considered as a set, as it has no multiplicity. Indeed, there is at most one way to complete a injection of inner vertices into a topological map from a given forest $F^{\prime}$ to a given forest $F$.

Lemma 4.2 Let $\sqcup$ and $\vee$ be the bilinear extensions of the operations $\sqcup$ and $\vee$ on forests.

1. Let $T=T_{1} \vee T_{2}$ be a tree and $V^{\prime}$ be a subset of $V(T)$ containing the bottom vertex $v$. Let $V_{1}^{\prime}=V^{\prime} \cap V\left(T_{1}\right)$ and $V_{2}^{\prime}=V^{\prime} \cap V\left(T_{2}\right)$. Then $\gamma\left(T, V^{\prime}\right)=\gamma\left(T_{1}, V_{1}^{\prime}\right) \vee \gamma\left(T_{2}, V_{2}^{\prime}\right)$.

2. Let $T=T_{1} \vee T_{2}$ be a tree and $V^{\prime}$ be a subset of $V(T)$ not containing the bottom vertex v. Let $V_{1}^{\prime}=V^{\prime} \cap V\left(T_{1}\right)$ and $V_{2}^{\prime}=V^{\prime} \cap V\left(T_{2}\right)$. Then $\gamma\left(T, V^{\prime}\right)=\gamma\left(T_{1}, V_{1}^{\prime}\right) \sqcup \gamma\left(T_{2}, V_{2}^{\prime}\right)$.

3. Let $F=F_{1} \sqcup F_{2}$ be a forest and $V^{\prime}$ be a subset of $V(F)$. Let $V_{1}^{\prime}=V^{\prime} \cap V\left(F_{1}\right)$ and $V_{2}^{\prime}=V^{\prime} \cap V\left(F_{2}\right)$. Then $\gamma\left(F, V^{\prime}\right)=\gamma\left(F_{1}, V_{1}^{\prime}\right) \sqcup \gamma\left(F_{2}, V_{2}^{\prime}\right)$.

Proof: The second and third cases are essentially the same and easy consequences of the definition of the poset. If two sets $V_{1}, V_{2}$ of inner vertices of a forest $F$ have no ancestor leaf in common, then the set $\gamma\left(F, V_{1} \sqcup V_{2}\right)$ is in bijection with the product $\gamma\left(F, V_{1}\right) \times \gamma\left(F, V_{2}\right)$. For $\gamma$ seen as a sum, this gives the expected result.

The first case now. Any element of $\gamma\left(T, V^{\prime}\right)$ is a forest $F$ with inner vertices $V^{\prime}$. This forest can be restricted to $V_{1}^{\prime}$ and to $V_{2}^{\prime}$ to give two forests $F_{1}$ and $F_{2}$. To be able to recover the forest $F$ from $F_{1}$ and $F_{1}$, it is necessary and sufficient to know to which tree of $F_{1}$ and to which tree of $F_{2}$ the vertex $v$ was connected in $F$. Therefore the set $\gamma\left(T, V^{\prime}\right)$ is in bijection with the set of quadruples $\left(F_{1}, \alpha, F_{2}, \beta\right)$ where $F_{1}$ and $F_{2}$ are in $\gamma\left(T_{1}, V_{1}^{\prime}\right)$ and $\gamma\left(T_{2}, V_{2}^{\prime}\right), \alpha$ is a tree of $F_{1}$ and $\beta$ is a tree of $F_{2}$.

Therefore, seen as a sum, $\gamma\left(T, V^{\prime}\right)$ is exactly given by the bilinear extension of the operation $\vee$ on forests, which is a sum over the set of pairs of subtrees.

\subsection{Explicit formula for the coproduct}

Proposition 4.3 Let $o \otimes F$ be an inner-oriented forest. Then

$$
\Delta(o \otimes F)=\sum_{V(F)=V^{\prime} \sqcup V^{\prime \prime}}\left(o^{\prime} \otimes \gamma\left(F, V^{\prime}\right)\right) \otimes\left(o^{\prime \prime} \otimes \gamma\left(F, V^{\prime \prime}\right)\right) .
$$

where the local orientations are unchanged and the global orientations satisfy $o^{\prime} \wedge r \wedge o^{\prime \prime}=o$ modulo $R^{\prime} \wedge r \wedge R^{\prime \prime}=R$. 


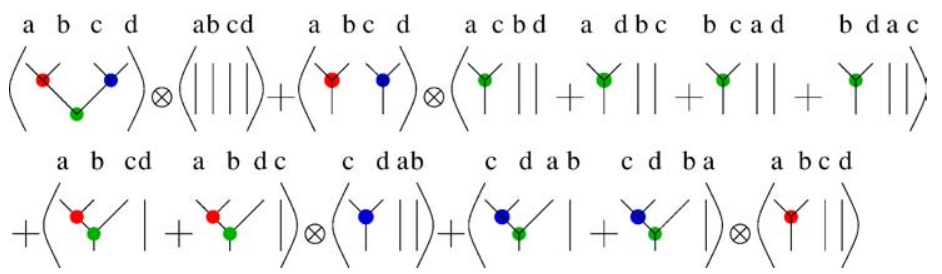

Figure 8. Half of an example for the cocommutative coproduct.

An example for this formula is given in figure 8, where only half of the terms are displayed because of cocommutativity, and where signs and orientations are omitted for simplicity.

Proof: The proof is a recursion on the number of inner vertices. The proposition is clear for trees with no inner vertex. The proof of the recursion step is done separately for trees and for forests with at least two trees.

The case of trees $\quad$ Let $o_{1} \otimes T_{1}$ and $o_{2} \otimes T_{2}$ be two inner-oriented trees and let $o \otimes T=$ $\left(o_{1} \vee o_{2}\right) \otimes\left(T_{1} \vee T_{2}\right)$. Then

$$
\begin{aligned}
\Delta(o \otimes T)= & \Delta\left(\Omega_{\star, \#} \circ_{\star}\left(o_{1} \otimes T_{1}\right){ }^{\circ_{\#}}\left(o_{2} \otimes T_{2}\right)\right) \\
= & \sum_{V\left(T_{1}\right)=V_{1}^{\prime} \sqcup V_{1}^{\prime \prime}} \sum_{V\left(T_{2}\right)=V_{2}^{\prime} \sqcup V_{2}^{\prime \prime}}\left(\Omega_{\star, \#} \otimes E_{\star, \#}+E_{\star, \#} \otimes \Omega_{\star, \#}\right) \\
& \rho_{\star}\left(o_{1}^{\prime} \otimes \gamma_{1}^{\prime} \otimes o_{1}^{\prime \prime} \otimes \gamma_{1}^{\prime \prime}\right) \circ_{\#}\left(o_{2}^{\prime} \otimes \gamma_{2}^{\prime} \otimes o_{2}^{\prime \prime} \otimes \gamma_{2}^{\prime \prime}\right),
\end{aligned}
$$

where $\gamma_{i}^{*}$ stands for $\gamma\left(T_{i}, V_{i}^{*}\right)$.

The first half of this formula corresponding to the expansion of the composition in $\Omega_{\star, \#} \otimes E_{\star, \#}$ is given by

$$
\begin{aligned}
& \sum_{V\left(T_{1}\right)=V_{1}^{\prime} \sqcup V_{1}^{\prime \prime}} \sum_{V\left(T_{2}\right)=V_{2}^{\prime} \sqcup V_{2}^{\prime \prime}}(-1)^{o_{2}^{\prime} o_{1}^{\prime \prime}}\left(\Omega_{\star, \#} \circ_{\star}\left(o_{1}^{\prime} \otimes \gamma_{1}^{\prime}\right) \circ_{\#}\left(o_{2}^{\prime} \otimes \gamma_{2}^{\prime}\right)\right) \\
& \otimes\left(E_{\star \star \#} \circ_{\star}\left(o_{1}^{\prime \prime} \otimes \gamma_{1}^{\prime \prime}\right) \circ_{\#}\left(o_{2}^{\prime \prime} \otimes \gamma_{2}^{\prime \prime}\right)\right) \\
& =\sum_{V\left(T_{1}\right)=V_{1}^{\prime} \sqcup V_{1}^{\prime \prime}} \sum_{V\left(T_{2}\right)=V_{2}^{\prime} \sqcup V_{2}^{\prime \prime}}(-1)^{o_{2}^{\prime} o_{1}^{\prime \prime}} \bar{o}^{\prime} \otimes\left(\gamma_{1}^{\prime} \vee \gamma_{2}^{\prime}\right) \otimes \bar{o}^{\prime \prime} \otimes\left(\gamma_{1}^{\prime \prime} \sqcup \gamma_{2}^{\prime \prime}\right),
\end{aligned}
$$

the orientations satisfying

$$
\begin{array}{rlrl}
o_{1}^{\prime} \wedge r_{1} \wedge o_{1}^{\prime \prime} & =o_{1} & R_{1}^{\prime} \wedge r_{1} \wedge R_{1}^{\prime \prime} & =R_{1} \\
o_{2}^{\prime} \wedge r_{2} \wedge o_{2}^{\prime \prime}=o_{2} & R_{2}^{\prime} \wedge r_{2} \wedge R_{2}^{\prime \prime}=R_{2} \\
(-1)^{o_{1}^{\prime}} o_{1}^{\prime} \wedge o_{2}^{\prime}=\bar{o}^{\prime} & R^{\prime} \wedge s=R_{1}^{\prime} \wedge R_{2}^{\prime} \\
o_{1}^{\prime \prime} \wedge r^{\prime \prime} \wedge o_{2}^{\prime \prime}=\bar{o}^{\prime \prime} & R_{1}^{\prime \prime} \wedge r^{\prime \prime} \wedge R_{2}^{\prime \prime}=R^{\prime \prime} .
\end{array}
$$


On the other hand, one has to compute

$$
\sum_{\substack{\left.V(T)=V^{\prime}\right\lrcorner V^{\prime \prime} \\ v \in V^{\prime}}} o^{\prime} \otimes \gamma\left(T, V^{\prime}\right) \otimes o^{\prime \prime} \otimes \gamma\left(T, V^{\prime \prime}\right)
$$

As $V(T)=\{v\} \sqcup V\left(T_{1}\right) \sqcup V\left(T_{2}\right)$, one can replace the sum by a double sum, using Lemma 4.2:

$$
\sum_{V\left(T_{1}\right)=V_{1}^{\prime} \sqcup V_{1}^{\prime \prime}} \sum_{V\left(T_{2}\right)=V_{2}^{\prime} \sqcup V_{2}^{\prime \prime}} o^{\prime} \otimes\left(\gamma_{1}^{\prime} \vee \gamma_{2}^{\prime}\right) \otimes o^{\prime \prime} \otimes\left(\gamma_{1}^{\prime \prime} \sqcup \gamma_{2}^{\prime \prime}\right),
$$

with the orientations determined by

$$
\begin{aligned}
o^{\prime} \wedge r \wedge o^{\prime \prime} & =o_{1} \vee o_{2} & R^{\prime} \wedge r \wedge R^{\prime \prime} & =R \\
(-1)^{o_{1}} o_{1} \wedge o_{2} & =o_{1} \vee o_{2} & R_{1} \wedge R_{2} & =R \wedge s .
\end{aligned}
$$

All these conditions on orientations together imply that the orientations $o^{\prime} \otimes o^{\prime \prime}$ and $(-1)^{o_{2}^{\prime} o_{1}^{\prime \prime}} \bar{o}^{\prime} \otimes \bar{o}^{\prime \prime}$ are the same. Therefore (19) and (20) are equal.

The other half of the sum (18), corresponding to the expansion of the composition in $E_{\star, \#} \otimes \Omega_{\star, \#}$, is shown in the same way to be equal to

$$
\sum_{\substack{\left.V(T)=V^{\prime}\right\lrcorner V^{\prime \prime} \\ v \in V^{\prime \prime}}} o^{\prime} \otimes \gamma\left(T, V^{\prime}\right) \otimes o^{\prime \prime} \otimes \gamma\left(T, V^{\prime \prime}\right)
$$

Therefore the full sum (18) is given by the expected formula (17) and the recursion step is done for trees.

The case of true forests Let $o_{1} \otimes F_{1}$ and $o_{2} \otimes F_{2}$ be two inner-oriented forests and let $o \otimes F=\left(o_{1} \sqcup o_{2}\right) \otimes\left(F_{1} \sqcup F_{2}\right)$. One has

$$
\begin{aligned}
\Delta & (o \otimes F)=\Delta\left(E_{\star, \#} \circ_{\star} o_{1} \otimes F_{1} \circ_{\#} o_{2} \otimes F_{2}\right) \\
= & \sum_{V_{1}=V_{1}^{\prime} \sqcup V_{1}^{\prime \prime}} \sum_{V_{2}=V_{2}^{\prime} \sqcup V_{2}^{\prime \prime}}\left(E_{\star, \#} \otimes E_{\star, \#}\right) \circ_{\star}\left(o_{1}^{\prime} \otimes \gamma_{1}^{\prime} \otimes o_{1}^{\prime \prime} \otimes \gamma_{1}^{\prime \prime}\right) \circ_{\#}\left(o_{2}^{\prime} \otimes \gamma_{2}^{\prime} \otimes o_{2}^{\prime \prime} \otimes \gamma_{2}^{\prime \prime}\right) \\
= & \sum_{V_{1}=V_{1}^{\prime} \sqcup V_{1}^{\prime \prime}} \sum_{V_{2}=V_{2}^{\prime} \sqcup V_{2}^{\prime \prime}}(-1)^{o_{2}^{\prime} o_{1}^{\prime \prime}}\left(E_{\star, \#} \circ_{\star} o_{1}^{\prime} \otimes \gamma_{1}^{\prime} \circ_{\#} o_{2}^{\prime} \otimes \gamma_{2}^{\prime}\right) \\
& \otimes\left(E_{\star, \#} \circ_{\star} o_{1}^{\prime \prime} \otimes \gamma_{1}^{\prime \prime} \circ_{\#} o_{2}^{\prime \prime} \otimes \gamma_{2}^{\prime \prime}\right) \\
& \sum_{V_{1}=V_{1}^{\prime} \sqcup V_{1}^{\prime \prime}} \sum_{V_{2}=V_{2}^{\prime} \sqcup V_{2}^{\prime \prime}}(-1)^{o_{2}^{\prime} o_{1}^{\prime \prime}} \bar{o}^{\prime} \otimes\left(\gamma_{1}^{\prime} \sqcup \gamma_{2}^{\prime}\right) \otimes \bar{o}^{\prime \prime} \otimes\left(\gamma_{1}^{\prime \prime} \sqcup \gamma_{2}^{\prime \prime}\right),
\end{aligned}
$$

where $\gamma_{i}^{*}$ stands for $\gamma\left(F_{i}, V_{i}^{*}\right)$ and the orientations satisfy

$$
\begin{array}{ll}
o_{1}^{\prime} \wedge r_{1} \wedge o_{1}^{\prime \prime}=o_{1} & R_{1}^{\prime} \wedge r_{1} \wedge R_{1}^{\prime \prime}=R_{1} \\
o_{2}^{\prime} \wedge r_{2} \wedge o_{2}^{\prime \prime}=o_{2} & R_{2}^{\prime} \wedge r_{2} \wedge R_{2}^{\prime \prime}=R_{2}
\end{array}
$$




$$
\begin{aligned}
o_{1}^{\prime} \wedge r^{\prime} \wedge o_{2}^{\prime}=\bar{o}^{\prime} & R_{1}^{\prime} \wedge r^{\prime} \wedge R_{2}^{\prime}=R^{\prime} \\
o_{1}^{\prime \prime} \wedge r^{\prime \prime} \wedge o_{2}^{\prime \prime}=\bar{o}^{\prime \prime} & R_{1}^{\prime \prime} \wedge r^{\prime \prime} \wedge R_{2}^{\prime \prime}=R^{\prime \prime} .
\end{aligned}
$$

On the other hand, one has to compute

$$
\sum_{V(F)=V^{\prime} \sqcup V^{\prime \prime}} o^{\prime} \otimes \gamma\left(F, V^{\prime}\right) \otimes o^{\prime \prime} \otimes \gamma\left(F, V^{\prime \prime}\right) .
$$

As $V(F)=V\left(F_{1}\right) \sqcup V\left(F_{2}\right)$, one can replace the summation by two separate summations, using Lemma 4.2:

$$
\sum_{V\left(F_{1}\right)=V_{1}^{\prime} \sqcup V_{1}^{\prime \prime}} \sum_{V\left(F_{2}\right)=V_{2}^{\prime} \sqcup V_{2}^{\prime \prime}} o^{\prime} \otimes\left(\gamma_{1}^{\prime} \sqcup \gamma_{2}^{\prime}\right) \otimes o^{\prime \prime} \otimes\left(\gamma_{1}^{\prime \prime} \sqcup \gamma_{2}^{\prime \prime}\right),
$$

with the orientations satisfying

$$
\begin{aligned}
o^{\prime} \wedge r \wedge o^{\prime \prime}=o_{1} \sqcup o_{2} & R^{\prime} \wedge r \wedge R^{\prime \prime}=R \\
o_{1} \wedge r_{12} \wedge o_{2}=o_{1} \sqcup o_{2} & R_{1} \wedge r_{12} \wedge R_{2}=R .
\end{aligned}
$$

One can then show by using all the conditions above that the orientations $o^{\prime} \otimes o^{\prime \prime}$ and $(-1)^{o_{2}^{\prime} o_{1}^{\prime \prime}} \bar{o}^{\prime} \otimes \bar{o}^{\prime \prime}$ are the same, which implies that (22) and (23) are equal. The recursion step is done for forests.

The proposition is proved.

Proposition 4.4 The projection to the one-dimensional degree zero component is a counit. The inclusion of this degree zero component is an augmentation.

Proof: For a finite set $I$, there is just one forest of degree zero, which has no inner vertex. By inspection of the formula for the coproduct, this forest is grouplike. The second part of the proposition follows. This forest can only be obtained in the coproduct of $F$ for the two summands given by $V(F) \sqcup \emptyset$ and $\emptyset \sqcup V(F)$, and the counit property is easily checked.

\section{Algebras of labeled binary trees}

As it is sometimes more convenient to work with algebras rather than coalgebras, we introduce here the algebra structure on the dual vector space of $\operatorname{Bess}(I)$. A finite set $I$ is fixed from now on. 


\subsection{Description and properties}

Let us consider the dual basis, still indexed by inner-oriented forests on $I$, of the dual vector space $\operatorname{Bess}^{*}(I)$, defined by the following pairing from $\operatorname{Bess}(I) \otimes \operatorname{Bess}^{*}(I)$ to $\mathbb{Q}$.

$$
\left\langle o \otimes F, o^{\prime} \otimes F^{\prime}\right\rangle=\left\{\begin{array}{ll}
0 & \text { if } F \neq F^{\prime}, \\
1 & \text { if } F=F^{\prime}
\end{array} \text { and } o=o^{\prime} .\right.
$$

The induced pairing from $\operatorname{Bess}(I) \otimes \operatorname{Bess}(I) \otimes \operatorname{Bess}^{*}(I) \otimes \operatorname{Bess}^{*}(I)$ to $\mathbb{Q}$ is denoted again by \langle\rangle .

It appears to be more convenient to use the opposite of the dual product.

Proposition 5.1 The opposite of the dual product is given by

$$
\left(o_{1} \otimes F_{1}\right) \times\left(o_{2} \otimes F_{2}\right)=\sum_{\left(F, V_{1} \sqcup V_{2}\right)} o \otimes F
$$

where the orientations satisfy $o_{1} \wedge r \wedge o_{2}=o$ and $R_{1} \wedge r \wedge R_{2}=R$, the sum being over the set of pairs $\left(F, V_{1} \sqcup V_{2}\right)$ where $F$ is a forest and $V(F)=V_{1} \sqcup V_{2}$ a partition of the set of inner vertices of $F$ such that $F_{1}$ appears in $\gamma\left(F, V_{1}\right)$ and $F_{2}$ appears in $\gamma\left(F, V_{2}\right)$.

Proof: The defining property of the dual product $x^{o p}$ is

$$
\left\langle o_{1} \otimes F_{1} \otimes o_{2} \otimes F_{2}, \Delta(o \otimes F)\right\rangle=\left\langle\left(o_{1} \otimes F_{1}\right) \times^{o p}\left(o_{2} \otimes F_{2}\right), o \otimes F\right\rangle .
$$

Let $\Delta(o \otimes F)=\sum o^{\prime} \otimes \gamma^{\prime} \otimes o^{\prime \prime} \otimes \gamma^{\prime \prime}$, with the orientations given by $o^{\prime} \wedge r \wedge o^{\prime \prime}=o$ and $R^{\prime} \wedge r \wedge R^{\prime \prime}=R$. The left hand-side of (26) can be computed as follows.

$$
\begin{aligned}
& \sum\left\langle o_{1} \otimes F_{1} \otimes o_{2} \otimes F_{2}, o^{\prime} \otimes \gamma^{\prime} \otimes o^{\prime \prime} \otimes \gamma^{\prime \prime}\right\rangle \\
& =\sum(-1)^{o^{\prime} o_{2}}\left\langle o_{1} \otimes F_{1}, o^{\prime} \otimes \gamma^{\prime}\right\rangle\left\langle o_{2} \otimes F_{2}, o^{\prime \prime} \otimes \gamma^{\prime \prime}\right\rangle \\
& =(-1)^{o_{1} o_{2}} \delta_{o^{\prime}, o_{1}} \delta_{F_{1} \in \gamma^{\prime}} \delta_{o^{\prime \prime}, o_{2}} \delta_{F_{2} \in \gamma^{\prime \prime}},
\end{aligned}
$$

where $\delta_{F \in \gamma}$ is 1 if $F$ belongs to the set/sum $\gamma$ and else 0 , and the orientations are identified in an obvious way. Here was used the fact that the $\operatorname{sum} \gamma(F, V)$ is without multiplicity.

Therefore, as taking the opposite product exactly removes the sign $(-1)^{o_{1} o_{2}}$, one has

$$
\left\langle\left(o_{1} \otimes F_{1}\right) \times\left(o_{2} \otimes F_{2}\right), o \otimes F\right\rangle=\delta_{o^{\prime}, o_{1}} \delta_{F_{1} \in \gamma^{\prime}} \delta_{o^{\prime \prime}, o_{2}} \delta_{F_{2} \in \gamma^{\prime \prime}} .
$$

The proposition follows.

Let the support of a forest $F$, denoted by $\operatorname{Supp}(F)$, be the set of leaves which are not linked to the root by an edge, i.e. such that the path to the root contains at least one inner vertex. 
Lemma 5.2 Let $F$ be any forest appearing in the product of $F_{1}$ and $F_{2}$. Then $\operatorname{Supp}(F)=$ $\operatorname{Supp}\left(F_{1}\right) \cup \operatorname{Supp}\left(F_{2}\right)$.

Proposition 5.3 Let $F_{1}, F_{2}$ be two forests on I with disjoint supports. Then the only forest appearing in the product of $F_{1}$ and $F_{2}$ is the forest $F$ with $\operatorname{Supp}(F)=\operatorname{Supp}\left(F_{1}\right) \sqcup \operatorname{Supp}\left(F_{2}\right)$ which coincides with $F_{1}$ and $F_{2}$ on their respective support.

Proof: Any forest appearing in the product should have support the disjoint union of supports. The condition that $F_{1} \leq F$ implies that the number of inner vertices of $F$ which are linked to the support of $F_{1}$ is greater or equal than the number of inner vertices of $F_{1}$. The same is true for $F_{2}$ and its support. But the number of inner vertices of $F$ is the sum of those of $F_{1}$ and $F_{2}$, therefore there is equality and the proposition follows.

For any set $I$ containing $\{i, j\}$, let $Y_{i, j}$ be the element of $\operatorname{Bess}^{*}(I)$ corresponding to the forest with one inner vertex, with support $\{i, j\}$ and orientation as in figure 5.

Lemma 5.4 Let $F$ be a forest on I with $j \notin \operatorname{Supp}(F)$. Then the forests appearing in $F \times Y_{i, j}$ are exactly all forests obtained from $F$ by grafting a leaf $j$ to any edge in the path from $i$ to the root.

Proof: It is clear that each such forest do appear in the product. We need only to show that there are no others. The forests which appear should have a vertex with leaves $i$ and $j$ as ancestors. As $j$ do not belong to the support of $F$, this vertex should be added to $F$. It can only be added on an edge of the path from $i$ to the root.

\subsection{Some relations and open questions}

Let us introduce some notation. Let $\operatorname{LLL}(i, j, k, \ell)$ be the inner-oriented forest on any set $I$ containing $\{i, j, k, \ell\}$, which is defined on its support $\{i, j, k, \ell\}$ by the same orientations and tree as figure 9.

Let $\mathrm{Y}_{\mathrm{Y}} \mathrm{Y}(i, j, k, \ell)$ be the inner-oriented forest on any set $I$ containing $\{i, j, k, \ell\}$, which is defined on its support $\{i, j, k, \ell\}$ by the same orientations and tree as figure 10 .

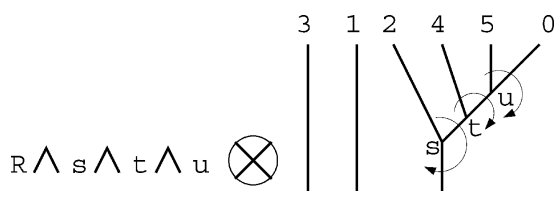

Figure 9. $\operatorname{LLL}(2,4,5,0)$ on $\{0,1,2,3,4,5\}$. 


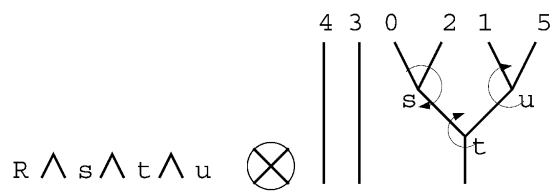

Figure 10. $\mathrm{Y}_{\mathrm{Y}} \mathrm{Y}(0,2,1,5)$ on $\{0,1,2,3,4,5\}$.

\section{Lemma 5.5 One has}

$\operatorname{LLL}(i, j, k, \ell)=-\operatorname{LLL}(i, j, \ell, k)$,

$\mathrm{Y}_{\mathrm{Y}} \mathrm{Y}(i, j, k, \ell)=-\mathrm{Y}_{\mathrm{Y}} \mathrm{Y}(i, j, \ell, k)$,

$\mathrm{Y}_{\mathrm{Y}} \mathrm{Y}(i, j, k, \ell)=\mathrm{Y}_{\mathrm{Y}} \mathrm{Y}(k, \ell, i, j)$.

The following relations are satisfied in any algebra Bess* $(I)$.

Proposition 5.6 Let $i, j, k$ be three distinct elements of I. Then

$$
Y_{i, j} \times Y_{j, k} \times Y_{k, i}=0 .
$$

Proof: There can be no forest with 3 inner vertices and support of cardinal 3. The proposition therefore follows from Lemma 5.2.

Remark that $Y_{i, j} \times Y_{j, k} \times Y_{k, \ell}=Y_{\ell, k} \times Y_{k, j} \times Y_{j, i}$.

Proposition 5.7 Let $i, j, k, \ell$ be four distinct elements of I. Then

$$
\sum Y_{i_{1}, i_{2}} \times Y_{i_{2}, i_{3}} \times Y_{i_{3}, i_{4}}=0
$$

where the sum is over the set of total orders on $\{i, j, k, \ell\}$ up to reversal.

Proof: Using the product rule for the orientations and Lemma 5.4, one computes

$$
\begin{aligned}
Y_{i, j} \times Y_{j, k} \times Y_{k, \ell}= & \operatorname{LLL}(i, j, k, \ell)+\operatorname{LLL}(i, \ell, j, k) \\
& +\operatorname{LLL}(\ell, i, k, j)+\operatorname{LLL}(\ell, k, j, i)+\mathrm{Y}_{\mathrm{Y}} \mathrm{Y}(i, j, k, \ell) .
\end{aligned}
$$

The sum of all 12 similar terms obtained from this one by permutations of $\{i, j, k, l\}$ is then seen to vanish, using the antisymmetry and symmetry properties of LLL and $Y_{Y} Y$ stated in Lemma 5.5.

It is an interesting open problem to give a presentation by generators and relations of the algebras $\operatorname{Bess}^{*}(I)$.

Question 1 Do the elements $Y_{i, j}$ generate Bess*(I)? 
Assuming an affirmative answer, one can then ask

Question 2 Do the relations above give a presentation of $\operatorname{Bess}^{*}(I)$ ?

\subsection{Differential forms and hyperplane arrangement}

Let $I$ be a finite set and $\mathbb{C}^{I}$ be the vector space with coordinates $\left(x_{i}\right)_{i \in I}$. Let $\mathrm{H}_{I}$ be the union of all hyperplanes $x_{i}-x_{j}=0$ for $i \neq j$ in the subspace $\sum_{i \in I} x_{i}=0$ of $\mathbb{C}^{I}$.

It is well known from the work of Cohen (see [3, 10]) that the Gerstenhaber operad is the homology of the little discs operad, whose underlying spaces are homotopy equivalent to the complements of the complex arrangements $\mathrm{H}_{I}$. Therefore, by the classical theorem of Arnold [1] computing the cohomology of this complement, the coalgebra associated to a finite set $I$ defined by the Hopf structure of the Gerstenhaber operad has the following description : it is isomorphic to the dual of the subalgebra generated by all forms $d\left(x_{i}-\right.$ $\left.x_{j}\right) /\left(x_{i}-x_{j}\right)$ for $i \neq j$ in $I$ inside the algebra of differential forms on the complement of $\mathrm{H}_{I}$.

The differential forms $Y_{i, j}=d\left(x_{i}-x_{j}\right) /\left(x_{i}-x_{j}\right)^{2}$ for $i \neq j$ in $I$ are defined on the complement of $\mathrm{H}_{I}$. Obviously, they satisfy $Y_{i, j}=-Y_{j, i}$.

Let $i, j, k$ be three distinct elements of $I$. Then one has clearly

$$
Y_{i, j} \wedge Y_{j, k} \wedge Y_{k, i}=0
$$

Further experimental evidence has been obtained showing that the algebra on forests of binary trees considered in this article should be isomorphic to a quotient of the subalgebra generated by the $Y_{i, j}$ inside the algebra of differential forms on the complement of $\mathrm{H}_{I}$.

\section{References}

1. V.I. Arnold, "The cohomology ring of the group of dyed braids," Mat. Zametki 5 (1969), 227-231.

2. F. Bergeron, G. Labelle, and P. Leroux, Combinatorial Species and Tree-Like Structures, Vol. 67 of Encyclopedia of Mathematics and Its Applications, Cambridge University Press, Cambridge, 1998. Translated from the 1994 French original by Margaret Readdy, With a foreword by Gian-Carlo Rota.

3. F.R. Cohen, "The homology of $\mathcal{C}_{n+1}$-spaces, $n \geq 0$," in The Homology of Iterated Loop Spaces, Vol. 533 of Lecture Notes, Springer-Verlag (1976) pp. 207-351.

4. V. Ginzburg and M. Kapranov, "Koszul duality for operads," Duke Math. J. 76(1) (1994), $203-272$.

5. E. Grosswald, Bessel Polynomials, Springer, Berlin (1978).

6. H.L. Krall and O. Frink, "A new class of orthogonal polynomials: The Bessel polynomials," Trans. Amer. Math. Soc. 65 (1949), 100-115.

7. M. Markl, "Distributive laws and Koszulness," Ann. Inst. Fourier (Grenoble) 46(2) (1996), 307-323.

8. M. Markl, S. Shnider, and J. Stasheff, Operads in Algebra, Topology and Physics, American Mathematical Society, Providence, RI (2002).

9. J.P. May, "Definitions: Operads, algebras and modules," in Operads: Proceedings of Renaissance Conferences (Hartford, CT/Luminy, 1995), Providence, RI, Amer. Math. Soc. (1997), pp. 1-7.

10. A.A. Voronov, "Homotopy Gerstenhaber algebras," in Conférence Moshé Flato 1999, Kluwer Acad. Publ., Dordrecht, Vol. II (Dijon) (2000), pp. 307-331. 